\title{
Research on Generation of Fractal Architecture Model Scheme via Iterated Function System (IFS)
}

\author{
Yong Sop $\mathrm{U}^{1,}{ }^{4}$, YU Ying ${ }^{3}$, HAN Dong ${ }^{2 *}$, SUN Jizhou ${ }^{1}$ \\ 1. School of Computer Science and Technology Tianjin University; 2. School of Computer Software Tianjin University \\ 3. School of Art Engineering Tianjin Vocational Institute; 4. University of Science, Pyongyang, D. P. R. of Korea; \\ Tianjin 300072, China \\ E-mail: 379201586@qq.com
}

\begin{abstract}
Able to directly study the internal rule by starting from the complicated phenomenon, fractal theory can describe complicated phenomenon in natural world. Nowadays, in-depth study and exploration are conducted for fractal in almost all domains of science; some architects have paid attention to it in contemporary architecture design and gained some enlightenment. In the current parametric architecture design, only computer programming means is introduced into traditional architecture design, and various complicated forms that surpass the traditional and modern buildings will be presented before us. Its development has drawn more and more attention. Based on the latest nonlinear mathematical theory of fractal geometry, this paper generates multiple 3D model prototypes for selection by applying nonlinear IFS, and further processes the models into design schemes of fractal architecture. This paper hopes to provide a new design thought and means for architecture design.
\end{abstract}

Keywords-Iterated Function System (IFS); Fractal Architecture; Attractor; Self-similarity; Parametric Design

\section{INTRODUCTION}

New thought is formed in the modern society gradually with scientific thinking development. Such change is influencing the development direction of fields like science, art and architecture. Fractal was defined and developed by B. B. Mandelbrot of IBM in 1975. It demonstrates the hidden rule of indecipherable chaotic phenomena and irregular forms via a new tool, and thus obtains a set of essential relationships between the part and entirety. [5] From the perspective of architecture design, the differences of architectural styles in different eras reflect the changes of design thinking in various eras. [3] Due to comprehension about fractal, contemporary architects desire to design architecture works with fractal concept, so as to reflect the beauty and diversity of nature. With development of such thought, fractal theory has become a starting point for new thought of architects. [1, 3, 4] From the angle of architecture, definition of space can be understood as "work of deciding the boundary". In cities, communities present fragmentation and differentiation, and require mobility and diversity. Due to the form of architecture, people can hardly distinguish its boundary. The difference between the entirety and individual is dimmed via flowing organization system. In another word, their form is complicated and has natural attributes.
Charles Jencks proposed "new paradigm in architecture" and made a comparative analysis for complexity of architecture design. Robert Ventri considered that complexity was the contrary concept of simplicity. In another word, complexity refers to the building character with rich attaching levels.

With popularization of parametric architecture design, designers are able to introduce and select fractal theory at a quantitative level.

By starting from such new thought, the architect Peter Eisenman proposed "fractal scaling" in 1980, and completed the work of "House 11".

The combination of fractal theory and architecture design has affected and changed people's cognition mode and habit about nature and cities by breaking the limitation of architecture aesthetics. A possibility of a brand-new thinking mode is provided for construction and development of modern cities. Based on study and derivation of IFS, this paper generates a fractal model by applying IFS. It is further developed into design scheme of fractal architecture.

\section{SELF-SIMILARITY OF FRACTAL AND IFS PRINCIPLE}

Self-similarity means that the entire part has some similarities with the internal part. In another word, the entirety and part as well as part and its local part resemble each other. Fig. 1 and Fig. 2 show the typical self-similarity sets. Fractal has provided a new perspective for solving limit problems. It will help us understand the concept of limit from a developing and brand-new angle. On the one hand, fractal can visualize limit by utilizing the feedback process; on the other hand, some fractal pictures will display the self-similarity with a perfect form.

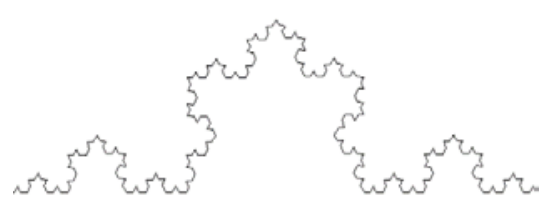

Fig. 1. Self-similarity set of Koch curve 


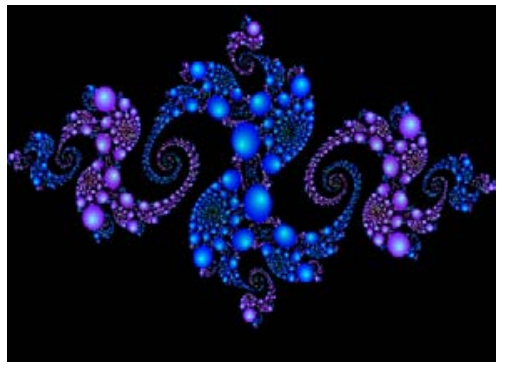

Fig. 2. Self-similarity set of Julia

Iterated function system (IFS) was founded by M. M. Bansley. As a basic direction of fractal theory study, it is in an in-depth study process. As for modeling based on IFS in this paper, IFS principle should be discussed at first.

\section{A. Basic principle of IFS and attractor of IFS}

[Theorem] Suppose that $\left\{X ; w_{i}: X \rightarrow X, i=1,2, \cdots N\right\}$ is hyperbolic IFS and there is a compressibility factor $S, W$ can be defined on $(H(X), h(d)) \cdot W: H(X) \rightarrow H(X)$,

$$
W(B)=\bigcup_{i=1}^{N} w_{i}(B), \forall B \in H(X)
$$

It is also the compressed mapping for $B \in H(X)$; there is a compressibility factor $S$,

$$
h(W(B), W(C)) \leq \operatorname{sh}(B, C)
$$

For $B, C \in H(X)$. There is a unique fixed point $A$ in $H(X)$ :

$$
A=W(A)=\bigcup_{i=1}^{N} w_{i}(A)
$$

$A$ Can be gained through the following formula: for $\forall$ $B \in H(X)$

$$
A=\lim _{n \rightarrow \infty} W^{n}(B)
$$

A Is known as attractor of IFS.

In IFS, continuous and tiny change of a parameter will result in continuous and tiny change of its attractor form. Therefore, different "copies" of the original image required in the coding process of generating fractal object can smoothly interpolate value among attractors.

\section{GENERATION OF 3D FRACTAL MODEL VIA IFS}

\section{A. Construction of nonlinear IFS}

The existing 2D fractal theory is utilized to extend to 3D fractal. In addition, nonlinear transformation is studied on the basis of linear transformation, and smooth transition is realized for the model via interpolation technology. Here a kind of new nonlinear IFS is constructed. By applying fractal interpolation, fractal interpolation curved surface is built through the given data points.
Let

$$
\begin{aligned}
& 0=x_{0}<x_{1}<\cdots<x_{M}=1, \\
& 0=y_{0}<y_{1}<\cdots<y_{N}=1, \\
& \left\{\left(x_{i}, y_{j}, z_{i j}\right) \mid i=0,1,2, \cdots, M, j=0,1,2, \cdots, N\right\}
\end{aligned}
$$

Given data

$w_{i j}:[0,1] \times[0,1] \times \mathbb{R} \rightarrow\left[x_{i-1}, x_{i}\right] \times\left[y_{j-i}, y_{j}\right] \times \mathbb{R}$ is defined by

$$
\begin{gathered}
w_{i j}\left(\begin{array}{l}
x \\
y \\
z
\end{array}\right)=\left(\begin{array}{c}
a_{i j} x+b_{i j} \\
c_{i j}+d_{i j} \\
e_{i j} x+f_{i j} y+\frac{1}{\sqrt{2}} g_{i j} \sqrt{x^{2}+y^{2}}+s_{i j} z+k_{i j}
\end{array}\right) \\
w_{i j}(i=1,2, \cdots, \quad M, j=1,2, \cdots, N)
\end{gathered}
$$

Suppose that $w_{i j}(i=1,2, \cdots, M, j=1,2, \cdots, N)$ is the qualified boundary condition

$$
\begin{array}{cc}
w_{i j}\left(\begin{array}{c}
x_{0} \\
y_{0} \\
z_{0,0}
\end{array}\right)=\left(\begin{array}{c}
x_{i-1} \\
y_{j-1} \\
z_{i-1, j-1}
\end{array}\right) & w_{i j}\left(\begin{array}{c}
x_{0} \\
y_{N} \\
z_{0, N}
\end{array}\right)=\left(\begin{array}{c}
x_{i-1} \\
y_{j} \\
z_{i-1, j}
\end{array}\right) \\
w_{i j}\left(\begin{array}{c}
x_{M} \\
y_{0} \\
z_{M, 0}
\end{array}\right)=\left(\begin{array}{c}
x_{i} \\
y_{j-1} \\
z_{i, j-1}
\end{array}\right) & w_{i j}\left(\begin{array}{c}
x_{M} \\
y_{N} \\
z_{M, N}
\end{array}\right)=\left(\begin{array}{c}
x_{i} \\
y_{j} \\
z_{i, j}
\end{array}\right)
\end{array}
$$

By transforming $w_{i j}(i=1,2, \cdots, M, j=1,2, \cdots, N)$ 9 real numbers are given $a_{i j}, b_{i j}, c_{i j}, d_{i j}, e_{i j}, f_{i j}, g_{i j}, s_{i j}, k_{i j}$. Moreover, these real numbers meet 8 linear equations.

$$
\begin{aligned}
& a_{i j} x_{0}+b_{i j}=x_{i-1} \\
& c_{i j} y_{0}+d_{i j}=y_{j-1} \\
& e_{i j} x_{0}+f_{i j} y_{0}+\frac{1}{\sqrt{2}} g_{i j} \sqrt{x_{0}^{2}+y_{0}^{2}}+s_{i j} z_{0,0}+k_{i j}=z_{i-1, j-1} \\
& c_{i j} y_{N}+d_{i j}=y_{j} \\
& e_{i j} x_{0}+f_{i j} y_{N}+\frac{1}{\sqrt{2}} g_{i j} \sqrt{x_{0}^{2}+y_{N}^{2}}+s_{i j} z_{0, N}+k_{i j}=z_{i-1, j} \\
& a_{i j} x_{M}+b_{i j}=x_{i} \\
& e_{i j} x_{M}+f_{i j} y_{0}+\frac{1}{\sqrt{2}} g_{i j} \sqrt{x_{M}^{2}+y_{0}^{2}}+s_{i j} z_{M, 0}+k_{i j}=z_{i, j-1} \\
& e_{i j} x_{M}+f_{i j} y_{N}+\frac{1}{\sqrt{2}} g_{i j} \sqrt{x_{M}^{2}+y_{N}^{2}}+s_{i j} z_{M, N}+k_{i j}=z_{i, j}
\end{aligned}
$$

The above iterated function system has the following characteristics:

- Not all attractors of nonlinear iterated function systems have continuity characteristic, but iterated function 
system of this study can guarantee boundary continuity during fractal interpolation. Compared with the existing iterated function systems, this system has greatly improved naturalness of interpolation.

- Extensive application scope: There is no limitation for size and symmetry of initial data set.

- The fractal interpolation curved surface can not only maintain major characteristics of original data in the whole but also possess self-similarity traits in local parts. Therefore, this iterated function system is more beneficial to describing practical application of natural object.

In the following studies, this paper plans to combine such IFS algorithm with computer application software, to create some 3D fractal models.

\section{B. Application of IFS to MATLAB software, to generate $3 D$ fractal models}

Iterated function system established in the above is interpolation function family; in another word, different 3D fractal models will be generated by importing different databases. At application level, this iterated function system can generate complex marginal models, such as mountain terrain and submarine topography. These complex marginal models can be further processed with modeling tools and developed into architecture model design schemes. At the first stage, some 3D fractal models are generated by applying IFS and MATLAB application software.

- Step1: Formulate MATLAB code;

- Step2: Write out database and save it as txt format;

- Step3: Import database (*.txt format) and give parameters $S_{i j}$

The code results are displayed through such algorithm.

TABLE I. TABLE FOR GENERATION OF 3D FRACTAL

\begin{tabular}{|l|l|l|}
\hline & \multicolumn{1}{|c|}{ Database } & \multicolumn{1}{c|}{ Parameter } \\
\hline Result 1 & mc & 0.01 \\
\hline Result 2 & mc & 0.2 \\
\hline Result 3 & mc & 0.9 \\
\hline Result 4 & ms & 0.01 \\
\hline Result 5 & ms & 0.3 \\
\hline Result 6 & ms & 0.7 \\
\hline
\end{tabular}

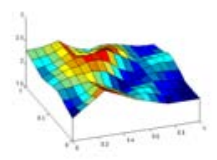

Result 1

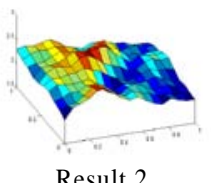

Result 2

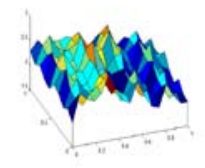

Result 3
Identify applicable sponsor/s here. If no sponsors, delete this text box (sponsors).

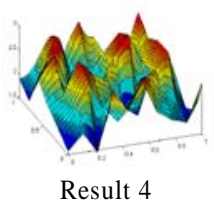

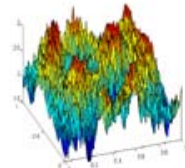

Result 5

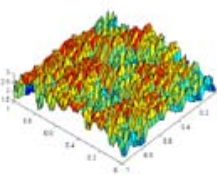

Result 6
Fig. 3. Different forms are generated with the change of $S_{i j}\left(0<S_{i j}<1\right)$ in iterated function system.

According to the above results, due to fractal theory of this paper, we have gained a new cognition about nature. The system can truly describe the ubiquitous objects in natural world, including irregular and rough (natural) scenes like mountain range and submarine topography. Such intuitional object description cannot be simulated by Euclidean geometry.

\section{APPLICATION OF IFS IN FRACTAL ARCHITECTURE MODEL}

\section{A. Discussion on application of IFS}

The above 3D fractal is generated through database and nonlinear transformation. In another word, the base material generated is database. After experiencing such IFS algorithm and thought, base material of IFS is replaced by base model (such as sphere and cuboid). Simply speaking, IFS is the ultimate complicated form after the initial unit modules undergo iterative operation on the basis of given transformation, including basic transformation sets like movement, rotation and compression. Even if simple image is set as the initial unit module, fractal forms close to natural features can be generated via IFS algorithm. Controllable model results that can be used for development will be gained on the basis of this algorithm. Finally, these models will develop into usable methods and tools of architecture design.

\section{B. Construction of $3 D$ configuration prototype via IFS}

Result of a form is controlled and adjusted by multiple parameters. In fractal, change of parameters can often develop into unexpected result and such result can even surpass the scope of human thinking. This is the charm of parametric design. In order to construct fractal model, this paper uses the 3D fractal establishment tool INCENDIA. 3D fractal model of IFS is completed in this software, and the results will be imported into 3d max software for operation. Besides basic transformation like movement, rotation and zooming, affine transformation is adopted. Algorithm and operation of model establishment are illustrated in the following. Affine transformation and 4 iterations are conducted for a regular tetrahedron, and then auxiliary parameters are adjusted. Different models are gained via this method.

Affine transformation:

$$
\left\{\begin{array}{l}
x(n+1)=a x(n)+b y(n)+c z(n)+j \\
y(n+1)=d(n)+e y(n)+f z(n)+k \\
z(n+1)=g x(n)+h y(n)+i z(n)+l
\end{array}\right.
$$


TABLE II. IFS CODES OF MODELS

\begin{tabular}{|l|l|l|l|l|}
\hline Parameter & \multicolumn{1}{|c|}{ Model 1 } & \multicolumn{1}{|c|}{ Model 2 } & \multicolumn{1}{|c|}{ Model 3 } & \multicolumn{1}{|c|}{ Model 4 } \\
\hline a & 0.91608 & 0.60432 & 0.71027 & 0.76458 \\
\hline b & 0.00693 & -0.05371 & 0.25832 & -0.38091 \\
\hline c & -0.12842 & -0.19608 & -0.10840 & -0.17658 \\
\hline d & -0.05321 & 0.02108 & -0.23036 & 0.39099 \\
\hline e & 0.86135 & 0.52251 & 0.70668 & 0.77965 \\
\hline f & -0.33315 & 0.12219 & 0.17466 & 0.01115 \\
\hline g & 0.11708 & 0.20220 & 0.15943 & 0.15296 \\
\hline h & 0.33730 & 0.21176 & -0.12977 & -0.08893 \\
\hline i & 0.85338 & 0.69572 & 0.73533 & 0.85413 \\
\hline j & 1.01783 & 0.21087 & -1.78006 & -1.07074 \\
\hline k & -0.52733 & 1.08862 & 0.75778 & -0.96659 \\
\hline l & 1.63889 & 1.66446 & 0.50710 & -1.38536 \\
\hline $\begin{array}{l}\text { Number of } \\
\text { iterations }\end{array}$ & 4 & 3 & 4 & 4 \\
\hline
\end{tabular}

A brief description is given to the generation process.
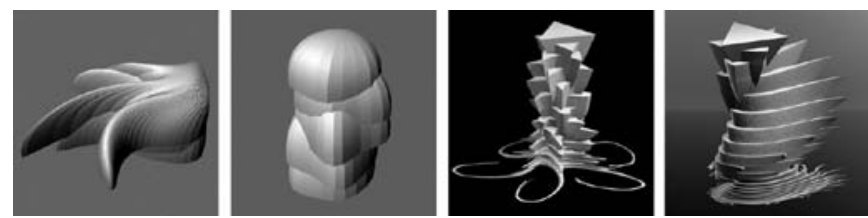

Fig. 4. 3D fractal model results generated with the change of IFS function (model 1, 2, 3 and 4 from left to right)

The above model results have multiple self-similarity structures. Their complexity represents the universality of most objects in natural world. When modeling is conducted by utilizing a basic model and adjustment parameter, unexpected and variable results can be discovered.

\section{Scheme design applied to fractal architecture model}

By further processing the models generated via IFS, the possibility of its application to fractal architecture design is revealed. (Note: This paper does not involve limitation of various environmental conditions in architecture design, such as limitations in foundation environment, structural material, and regional culture space; generation and processing of fractal architecture forms are discussed.) Fractal model result 1 gained in the above is used as prototype of the form. The model is deeply processed, rendered and output in virtue of the 3D graphic software 3DMAX, so as to complete fractal architecture design schemes (see Fig. 5).

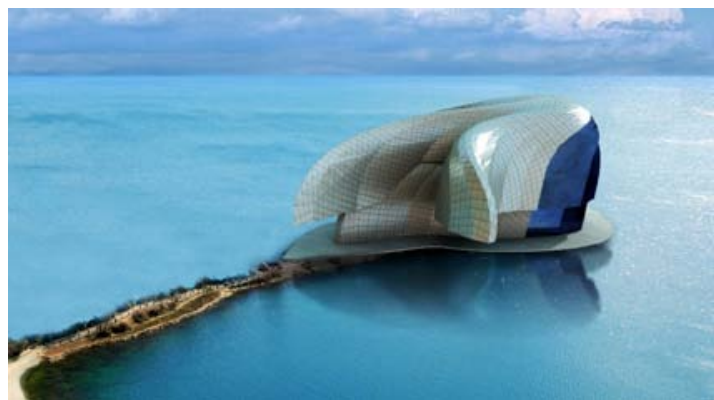

Fig. 5. Fractal architecture design schemes based on IFS

\section{CONCLUSION}

In this paper, method and algorithm of constructing fractal architecture models are discussed, and a nonlinear iterated function system is applied to fractal architecture model design. A logical relationship is established among transformation parameters of the form, other parameters and architecture forms, so as to gain the prototype of fractal architecture form with unpredictability through parametric settings. Later the architecture model is further processed with the help of 3D software like 3DMAX, and usable fractal architecture design schemes are formed. Our research achievement has obvious application possibility and presents the following characteristics. Firstly, in order to complete architecture model design based on IFS principle and algorithm, a new nonlinear iterative function is constructed. The experiment result proves the effectiveness and complexity of this system. Secondly, generation and practice of fractal architecture model can realize parametric design and return to natural logic and depth. Moreover, the design thought can be expanded and new forms will be presented. Thirdly, by applying fractal theory and iterated function to architecture design field, more extensive research space and effective tools will be provided for this field.

\section{REFERENCES}

[1] Carl Bovil, Fractal Geometry in Architecture and Design, Boston, Birkhauser, 1996

[2] Ron Eglash African Fractals, New Jersey, Rutgers University Press, 1999

[3] James Harris, Fractal Architecture Organic Design Philosophy in Theory and Practice, Twin, UNM Press, 2012

[4] M. Batty, P. Longly, Fractal Cities, London: Academic Press, 1994

[5] M.Bansley, Fractal Everywhere, New York, Academic Press, 1988

[6] T. Martyn "Realistic rendering 3D IFS fractals in real -time with graphics accelerators", Chaos and Graphics, vol. 34(3), pp. 167-175, 2010

[7] YAN QIU CHEN, "GUOAN BI.3-D IFS FRACTALS AS REAL-TIME GRAPHICS MODEL”, Chaos and Graphics, vol. 21(3), pp. 367-370, 1997

[8] Bourke P. "Evaluating Second Life for the collaborative exploration of 3D fractals”, Computers \& Graphics, vol. 33(1) pp. 113-117, 2009

[9] Nikiel S. Iterated function systems for real-time image synthesis, London, Springer, 2007

[10] Falconer K. Fractal geometry, 2nd ed. Chichester, Wiley, 2003 